\title{
Ueber die Unterphosphorsäure ;
}

\section{von Th. Salzer.}

(Eingelaufen den 5. Marz 1877.)

(Hierzu Tafel III, Figur 1 bis 4.)

Erste A b ba nd l u n g.

Wird Phosphor bei Gegenwart von Wasser so lange der Luft ausgesetzt, bis er möglichst oxydirt ist, d. h. bis die Flüssigkeit aus der Luft keinen Sauerstoff mehr aufnimmt, so erhält man die sogenannte Pelletier'sche Sâure (Acide phosphatique), welche eine constante Sauerstoffmenge enthalten soll *). Es wurde deshalb diese Săure von verschiedenen Forschern als eine eigene 0xydationsstufe des Phosphors, von anderen als eine Verbindung von Phosphoroxyd oder von phosphoriger Sâure mit Phosphorsäure betrachtet. Die Salze, welche Fourcroy und $V$ a uquelin mittelst dieser Säure und Kali resp. Natron dargestellt haben (siehe $\mathrm{Gmelin}$ bei den betr phosphorigsauren Salzen), konnten den gesuchten Beweis nicht liefern, da sie nicht rein gewesen, auch später, wie es scheint, nicht mehr dargestellt worden sind.

Nach Thé nard enthält die Pelletier'sche Säure $47 \mathrm{pC}$. Phosphor und 53 pC. Sauerstoff; nach D u l ong 47,85 pC. Phosphor und 52,15 pC. Sauerstoff, woraus dieser die Formel $P_{8} O_{8}$ ableitete, welche 46,26 pC. Phosphor und 53,74 pC. Sauerstoff verlangt; die $\mathrm{D}$ ulong'scben Zablen würden übrigens eben so gut zu der Pormel PO, passen, welche $49,2 \mathrm{pC}$. Phosphor und 50,8 pC. Sauerstoff erfordert.

*) Siehe Gmelin-Krant's Handbuah, 6. Aufl. 1, 2. Abth. 118, bei phosphoriger stinre. 
$\mathrm{Zu}$ meinen Untersuchungen hat mir keine Pelletier'sche Săure zur Verfügung gestanden ; es ist mir aber gelungen nachzuweisen, dais bei der langsamen Oxydation des Phosphors neben phosphoriger Säure (welche dann allmälig in Phosphorsãure übergeht) auch Unterphosphorsäure .entsteht, d. h. eine Sãure, welche in ihrem Sauerstoffgehalt zwischen Phosphorsãure und phosphoriger Säure steht und aus der Luft keinen weiteren Sauerstoff mehr aufnimmt; es würde diefs allein schon hinreichen, um die Brscheinung zu erklären, dafs der Phosphor unter diesen Verhältnissen nicht vollständig zu Phosphorsăure oxydirt wird.

Vielleicht ist der D u long 'schen Formel nicht allzuviel Werth beizulegen, da die hier in Frage kommenden Differenzen in der procentischen Zusammensetzung nur sehr unbedeutend sind; sie ist aber ungezwungen als richtig anzunehmen, wenn die Pelleti er'sche Säure auf ein Atom Unterphosphorsăure immer je ein Atom Phosphorsāure enthält : $\mathrm{PO}_{1}+\mathrm{PO}_{5}=$ $P_{x} O_{9}$; es ist diefs jedoch nicht wahrscheinlich, da nach meinen Beobachtungen des Oxydationsprocesses die Bildung der phosphorigen Säure und der Phosphorsäure jene der Unterphosphorsäure überflügelt.

Bei der Einwirkung von Salpetersäure auf Phosphor verläuft der Oxydationsprocefs in anderer Weise (dessen muthmafslich einfachste Form durch die Gleichung $P+N \mathrm{C}_{\mathrm{s}}=$ $\mathrm{PO}_{3}+\mathrm{NO}_{4}$ in dem ersten Stadium ansgeu. ackt wird) und es scheint sich hierbei keine Unterphosphorsäure za bilden; wenigstens ist es mir bis jetzt nicht mỏglioh gewesen, aus den Producten der Binwirkung von Salpetersäure verschiedener Stärke und bei niederen wie höheren Temperaturen die Sãure abzuscheiden, was allerdings durch die gleichzeitige Gegenwart von Salpetersäure und salpetriger Säure jedenfalls sehr ersohwert wird; anderseits würde die Anwesenheit von 
Unterphosphorsäure die Schwierigkeit erklären lassen, mit welcher bei der üblichen Darstellungsweise der reinen Phosphorsäure die. Beendigung der Oxydation verknüpft ist; die Unterphosphorsäure läfst sich nämlich, wie ich später zeigen werde, durch Salpetersäure nicht direct in Phosphorsãure überführen.

Bei der Yerbrennung des Phosphors in atmosphärischer Luft wird keine bemerkbare Menge von Unterphosphorsäure gebildet, sie entsteht dagegen, wenn phosphorige Säure längere Zeit der Einwirkung der Luft ausgesetzt wird.

\section{Unterphosphorsäure.}

Der saure Syrup, welchen man erhält, wenn man Phosphorstücke, welche theilweise in Wasser eingetaucht sind, an der Luft zerfliefsen lärst, enthält phosphorige Säure, Phosphorsäure und Unterphosphorsäure. Die letztere läfst sich leicht in Form eines verhältnifsmäfsig schwerlöslichen sauren Natronsalzes durch Behandlung des Säuregemisches mit kohlensaurem oder essigsaurem Natron von den beiden anderen Säuren des Phosphors tiennen.

Die reine Unterphosphorsäure wird am besten aus frisch gefälltem, in Wasser suspendirtem reinem unterphosphorsaurem Bleioxyd durch Behandlung mit Schwefelwasserstoff dargestellt, indem aus der von dem gebildeten Schwefelblei abfiltrirten Flüssigkeit durch Aufkochen der überflüssige Schwefelwasserstoff leicht verjagt werden kann. Es ist dabei zu beachten, dafs die etwaigen Verunreinigungen des zur Fällung verwendeten Bleisalzes, wie z. B. Eisen, in den Bleiniederschlag übergehen und dann durch die Unterphosphorsäure wieder aufgelöst werden können; ebenso mufs auch das zur Darstellung dienende unterphosphorsaure Natron frei von den übrigen Säuren des Phosphors, wie des Schwefels, Arsens u. s. w. sein; es ist dieses Salz aber verhältnifsmäfsig leicht 
rein darzustellen, weil es durch seine Schwerlōslichkeit in kaltem Wasser vor den meisten übrigen Natronsalzen ausgezeichnet ist und leicht krystallisirt.

Die auf diese Weise erhaltene wässerige Lösung von Unterphosphorsäure ist eine stark saure, farb- und geruchlose Flüssigkeit, welche obme Nachtheil lãngere Zeit gekocht werden kann; will man sie jedoch bis zur Syrupconsistenz eindampfen, so darf gegen das Ende nur eine möglichst niedere Temperatur (ca. $30^{\circ}$ ) angewendet werden, indem sonst eine Zerlegung der Säure in phosphorige Säure und Phosphorsäure beginnt; bei stärkerem Erhitzen zerfällt dann auch die gebildete phosphorige Säure, wie bekannt, noch weiter unter Entwickelung von Phosphorwasserstoff und Bildung tines gelben Körpers, welcher phosphorsaures Phosphoroxyd sein soll.

Bin krystallisirtes Hydrat oder das Anhydrid der Unterphosphorsäure habe ich noch nicht darzustellen versucht, da es mir noch an dem nothwendigen Material mangelte.

In ihrem Verhalten gegen Reagentien steht die Unterphosphorsãure zwischen der phosphorigen Säure und der Phosphorsäure, wodurch es erklärlich wird, dafs dieselbe in Gemischen von beiden so lange übersehen wurde.

Die wãsserige Lōsung der Săure ist vollkommen luftbestāndig und wird auch von concentrirten Sãuren in der Kälte nicht verăndert; wird sie jedoch mit verdūnnter Schwefelsäure gekocht, so zerfällt bei einer gewissen Concentration die Säure in phosphorige und Phosphorsãure; merkwürdiger Weise verhält sich die Unterphosphorsãure gegen Salpetersäure ganz ähnlich : in verdūnntem Zustande wirken die beiden Säuren scheinbar nicht auf einander ein, selbst wenn die Flüssigkeit längere Zeit gekocht wird, und das Säuregemisch verhält sich gegen Reagentien, hier besonders gegen salpetersaure Silberlösung, nach wie vor; wird jedoch noch stärker eingedampft, so zerfâllt die Säure ebenfalls in phosphorige Sãure und 
Phosphorsaure, und es scheint, dafs die oxydirende Wirkung der Salpetersäure auf die freiwerdende phosphorige Săuro erst dann beginnt, nachdem alle Unterphosphorsäure zerstört ist.

Ich mufs hier jedoch bemerken, dafs während die verdünnte Unterphosphorsäure mit molybdänsaurem Ammoniak und Salzsäure weder in der Kälte noch in der Wärme den bekannten gelben Niederschlag giebt und dafs während die Flüssigkeit in der Kälte auch dann klar bleibt, wenn, wie üblich, die Molybdansäurelösung mittelst Salpetersãure angesãuert war, doch sofort ein gelber Niederschlag entsteht, wenn diese verdünnte Flüssigkeit erwärmt wird. Der Niederschlag enthält, wie aus seinem Verhalten gegen übermangansaures Kali hervorgeht, keine Unterphosphorsäure; ich kann diesen scheinbaren Widerspruch nur dadurch erklären, dafs die Gegenwart der Molyböänsäure die zersetzende Wirkung der Salpetersäure unterstützt, oder durch die ganz aufserordentliche Empîndlichkeit dieser Reaction.

Fügt man zu einer Flüssigkeit, welche Unterphosphorsãure und mit Salzsäure angesäuerte Molybdänsäurelösung enthält und welche beirn Erwärmen klar und farblos geblieben war, einige Tropfen Salpetersäure und erwärmt abermals, so entsteht der gelbe Niederschlag, dessen Menge zunimmt in dem Mafse, als Salpetersäure weiter zugefügt wird; ähnlich verhält sich die Flüssigkeit auf Zusatz von chlorsaurem Kali.

Wie gegen Salpetersäure, so verhält sich auch gegen die übrigen Oxydationsmittel die Unterphosphorsäure sehr indifferent, mit einziger Ausnahme des übermangansauren Kalis, welches merkwürdiger Weise auf phosphorige Säure nur sehr langsam einwirkt.

Durch Erwärmen mit verdünntem Wasserstoffsuperoxyd ${ }^{*}$ )

*) Ich will dabei nicht unerwahnt lassen, dafs das verwendete Wasserstoffsuperoxyd, welches aus Natriumsuperoxyd dargestellt war, 
wird die Unterphosphorsăure nicht oxydirt. Die Lōsungen von chromsaurem Kali, Chlor oder Jod in Jodkalium werden durch die Unterphosphorsãure auch bei Kochhitze nicht verändert; eben so wenig werden die Auflossungen von Quecksilberchlorid, Goldchlorid oder Chlorplatin reducirt oder gefällt. In Silberlösung erzeugt die Säure einen weifsen Niederschlag, wolcher bei Kochhitze nicht geschwärzt wird. Der Niederschlag entsteht selbst dann, wenn die Flüssigkeit einige freie Schwefelsäure oder Salpetersăure enthălt und das gefällte unterphosphorsaure Silberoxyd ist gegen das Licht ziemlich unempfindlich; es löst sich in einer grötseren Menge Salpetersăure und in Ammoniak leicht auf; die ammoniakalische Lösung wird durch Kochen nicht geschwärzt.

Hierdurch ist die Unterphosphorsäure leicht von der phosphorigen und unterphosphorigen, sowie von der gewöhnlichen Phosphorsäure zu unterscheiden; aulserdem ist ihr Verhalten jegen übermangansaures Kali sehr charakteristisch, indem dessen Lösung in der Kälte nur langsam, sehr rasch aber beim Erwärmen durch die mit Schwefelsăure versetzte Auflösung der Unterphosphorsäure entfärbt wird, während nach Rose die phosphorige Säure durch Chamäleon selbst bei Kochhitze nur sehr langsam oxydirt werden soll.

Das Product der Oxydation von Unterphosphorsäure mittelst übermangansauren Kali's ist die gewōhnliche Phosphorsäure.

Auch gegen Reductionsmittel ist die Unterphosphorsāure sehr beständig, indem Schwefelwasserstoff, schweflige Sāure,

sich durch grofse Bestandigkeit ansseichnet und gekocht werden kann, ohne zu zerfallen. Nach vierzehntlgiger Aufbewahrung zerlegte es noch eben so viel Chamaleon, wie zuror. (Diese Bestlandigkeit des Wasserstofisaperoxyds wuxde schon beobschtet von Böttger, Jahresbericht für Chemie u. 8. w. f. 1873, 188. D. R.) 
eine Mischung von Zink und Schwefelsāure olne Einwirkung auf ihre wässerige. Lösung sind.

Ueber das Verhalten der Unterphosphorsäure gegen die übrigen Reagentien erwähne ich noch Folgendes :

In einer kalt gesättigten Auflōsung von essigsaurem $\mathrm{Na}$ tron erzeugt die Säure einen krystollinischen Niederschlag, welcher unter dem Mikroscop in Form von mchr ader weniger regelmäfsigen sechseckigen Tafeln oder in feinen Nadeln erscheint, je nachdem zu seiner Entstehung längere oder kürzere Zeit erforderlich war. Chlornatriumlösung wird nicht getrü̉ıt, Kalkund Barytwasser werden weifs, Chlorcalcium- und Chlorbaryumlösung aber nicht gefällt; in einer Auflösung von schwefelsaurer Magnesia entsteht erst dann ein Niederschlag, nachdem Ammoniak zugefügt worden. Auf genügenden Zusatz von Salmiak verschwindet der Niederschlag wieder; ebenso werden Alaunund schwefelsaure Zinklösung erst auf Zusatz von Ammoniak gefällt und der erzeugte Niederschlag ist in überschüssigem Ammoniak löslich. In schwefelsaurer Eisenoxydullösung entsteht ein geringer, in Eisenchloridlösung ein reichlicher weifser Niederschlag.

Die Lösungen des essigsauren wie des salpetersauren Bleioxyds werden von der Säure weifs gefällt ; der in Kupfervitriollösung erzeugte bläuliche Niederschlag verändert seine Farbe bei Kochhitze nicht. Während Quecksilberchlorid nicht gefällt wird, entstehen in der salpetersauren Quecksilberoxydwie -oxydullösung weifse Niederschläge, die durch Kocinen keine Farbenveränderung erleiden.

Die Salze der Unterphosphorsäure verhalten sich im Allgemeinen ähnlich denen der phosphorigen und der unterphosphorigen Säure, sind aber doch leicht von ihnen mittelst Silberlösung und Chamäleon zu unterscheiden; sie sind aufserdem viel beständiger als diese, werden aber bei höherer Temperatur eben so zersetzt, indem reiner Wasserstoff oder Phos- 
phorwasserstoff entweicht und Phosphormetall oder phosphorsaures Salz oder Gemische von beiden zurückbleiben; diese Art der Zersetzung ist natürlich nur dann möglich, wenn das Salz Krystall- oder Constitutionswasser enthält, anderenfalls kōnnen die Salze, wie z. B. das Bleisalz, auch einfach durch Aufnahme von Sauerstoff in phosphorsaure Salze übergehen.

In Nachstehendem gebe ich die Reactionen der Natronsalze, soweit sie von den Reactionen der Säure bemerkenswerth verschieden sind.

Platin-, Gold- und Quecksilbercblorid werden auch von den Natronsalzlösungen nicht gefällt.

In einer Auflösung von schwefelsaurer Magnesia erzeugt das neutrale unterphosphorsaure Natron sogleich einen krystallinischen Niederschlag, während das saure unterphosphorsaure Natron in der Kälte keine Fällung hervorruft; erst beim Erwärmen entsteht ein pulveriger Niederschlag, welcher beim Erkalten theilweise wieder verschwindet, auch in Salmiak vollständig löslich ist. Enthält die Magnesialösung bereits Chlorammonium, so entsteht auch bei Kochhitze keine bleibende Trübung. Giefst man umgekehrt die Magnesialösung in die Auflōsung des sauren unterphosphorsauren Natrons, so hindert die Gegenwart von Chlorammonium die Entstebung des Niederschlags nicht.

Chlorbaryum wird schon durch die Auflosung des sauren unterphosphorsauren Natrons gefällt; die Menge des Niederschlags wird aber durch Kochen und noch mehr durch $\mathrm{Zu}$ satz von Ammoniak vergröfsert.

Alaun- und Zinkvitriollösungen bleiben anfangs kiar, aber nach einiger Zeit entstehen gelatinöse Niederschläge.

Schwefelsaures Eisenoxydul wird weifslich, Eisenchlorid gelblichweifs gefält. In der Auflösung des salpetersauren Kobaltoxyduls erzeugt das neutrale Salz sofort, das saure erst 
nach längerer Zeit oder beim Erwärmen röthliche Niederschläge; das schwefelsaure Cadmiumoxyd wird weifs gefällt.

Die Zusammensetzung der wasserfrei gedachten Unterphosphorsäure entspricht der Formel :

$P O_{1}$.

Da die Sãure zweibasisch ist, erhält das Hydrat die Formel :

$$
\mathrm{PO}_{4} .2 \mathrm{HO} \text { oder } \mathrm{PO}_{8} \mathrm{H}_{4} \text {. }
$$

Um die Zusammensetzung der Säure zu bestimmen ist es nicht gerade nöthig, die Säure selbst darzustellen, man kann auch das Natronsalz dazu verwenden; es ist nämlich, wie schon oben bemerkt, sehr schwierig, die Säure durch Einwirkung von Salpetersäure vollständig in gewöhnliche Phosphorsäure überzuführen und es fehlt an jedem äufserlich wahrnehmbaren Kriterium, ob die Oxydation vollendet ist. Ich habe deshalb vorgezogen, die Sauerstoffaufnahme durch übermangansaures Kali zu bestimmen, die gebildete Phosphorsäure mittelst Molybdänsäure von dem Kali, eventuell auch Natron und Mangan zu trennen, als phosphorsaure AmmoniakMagnesia zu fällen und endlich als pyrophosphorsaure Magnesia zu wägen.

10 CC. einer Lösung von Unterphosphorä̆ure rerlangten bei mehreren Versuchen 32,6 bis 32,8 CC. einer Chamkleonlösung, welohe auf den Liter 1,25 Grm. übermangansaures Kuli entinglt und von welcher zufolge Vergleichung mit Normaloxalsäurolösung 1 CC. 0,0003 Sanerstoff enteprach. Diese Menge Sànre bedurfte also zu ihrer vollständigen Oxydation 0,0098 Sauerstoff.

Die gebildete Phogphorsbure lieferte auf die oben angegebene Weise abgeschieden 0,140 pyrophosphorsaure Magnesis (welche allordings noch Spuren ron Molybdänoxyd enthielt), welchs 0,0391 Phosphor enthalten; 391 Th. Phosphor nehmen als: beim Uebergang von Unterphosphorsäure in Phosplorsäure noch $98 \mathrm{Th}$. Sauerstoff auf, oder ein Atom $=31 \mathrm{Th}$. Phosphor 7,77 Th. Bauerstoff, whlurend 1 Atom Sauerstoff 8 Theile verlangen würde.

Eine andere Bestimmung, bei weloher das Mangan mittelet Weinsteinsüure und Bchwefelammoniuon abgeschieden worden, iioferte 
eine kleine Menge pyrophosphorsanre Magnesia mehr $(0,147$ Grm. aus derselben Băuremenge).

Bei der Titration mittelst übermangansauren Kalis ist zu beachten, dafs dieses der mit Schwefelsäure versetzten und etwas erwärmten Flüssigkeit möglichst rasch zugefügt wird ; andernfalls würde man wegen des allmäligen Zerfallens der Unterphosphorsãure keine übereinstimmenden Zahlen erhalten. Zur Titerstellung der anzuwendenden sehr verdünnten übermangansauren Kadilösung benutzte ich $1 / 10^{-N o r m a l o x a l s a ̈ u r e-~}$ lösung.

Saures unterphosphorsaures Natron. $\mathrm{PO}_{4}, \mathrm{NaO}, \mathrm{HO}+6 \mathrm{HO}$ oder $\mathrm{P} \mathrm{\theta}_{3} \mathrm{NaH}+3 \mathrm{H}_{2} \mathrm{\theta}$.

Zur Darstellung dieses Natronsalzes, welches den Ausgangspunkt für die Bereitung der Unterphosphorsäure und ihrer Salze bildet, wird das durch Zerfliefsen von Phosphor an der Luft entstandene syrupöse Säuregemisch mit einer kalt gesāttigten Auflösung von essigsaurem Natron im Ueberschufs versetzt, d. h. bis durch ferneren Zusalz der Salzlösung die Menge des Niederschlags sich nicht mehr vergröfsert; wenn die Lösung verdünnt war, löst man geradezu die möglichst grofse Menge krystullisirtes essigsaures Natron darin auf. Es wird dann sofort oder nach längerer Zeit je nach dem Gehalt an Unterphosphorsäure ein reichlicher oder geringer Niederschlag entstehen, welcher durch Decantiren und Auswaschen auf einem Filter gereinigt und dann umkrystallisirt wird; ist die Krystallbildung sehr reichlich, so befördert man die $\mathbf{A b}$ scheidung aus der Flüssigkeit durch Verdünnen mit wenig Wasser.

Hat man gröfsere Mengen von Săuregemisch, so empfiehlt es sich, die nicht allzu concentrirte Flüssigkeit in zwei Theile zu theilen, den einen Theil mit kohlensaurem Natron zu neutralisiren (während dem zuweilen schon Salz auskrystallisirt) 
und dann den anderen Theil der Säure zuzumischen. Nach einigem Stehen wird das Salz sich in feinen Nadeln abscheiden; ist diefs jedoch nicht der Fall, so mufs die Flüssigkeit eingedampft werden, bis sie Krystalle liefert. Die syrupartige Mutterlauge wird mit essigsaurem Natron vermischt noch eine kleine Menge Salz liefern; sie enthält aufserdem nur unkrystallisirbare Lösungen von phosphorigsaurem und phosphorsaurem Natron in überschüssiger Säure.

Aus dem Gemisch der verschiedenen Oxydationsstufen des Phosphors scheidet sich das Natronsalz meistens nur in sehr geringer Menge und in wechselnden Formen ab; gewöhnlich erhielt ich zuerst feine federartige Krystalle, welche sehr viel Mutterlauge einschliefsen, dann nach deren Entfernung schön ausgebildete büschelig gruppirte Nadeln, welche endlich durch Umkrystallisiren das reine Salz liefern.

Die Form, in welcher sich das Natronsalz ausscheidet, ist durch die Concentration der Flüssigkeit bedingt. Läfst man verdünnte Lösungen längere Zeit stehen, so erscheinen Tafeln auch aus der Rohsaure. Das Salz krystallisirt in dem monoklinischen System und bildet schief rhombische Tafeln, welche an ihren scharfen Kanten häufig so stark abgestumpft sind, dafs scheinbar hexagonale sechsseitige Tafeln entstehen; häufig sind auch rinnenartig verwachsene Zwillingsformen bemerkbar.

Herr Professor K. Ha ush of er in München hatte die Güte, das Salz krystallographisch zu untersuchen; er giebt die nachstehende Beschreibung und die Zeichnungen Taf. III, Fig. 1 bis 4 :

\author{
„Klinorhombisch. \\ Axenverhältnifs a : b : c $=1,9964: 1: 2,0104$.
}

Neigung der Klinodiagonalen zur Verticalaxe $=\mathrm{C}=53^{\circ} 14^{\prime}$.

Beobach tete Flächen :

$\infty \mathrm{P}(\mathrm{P}), 0 \mathrm{P}(\mathrm{B}), \frac{\mathrm{P}}{2}(\mathrm{~S}), 2 \mathrm{P} \infty(\mathrm{O})$. 
Gemessene Winkel :

$$
\begin{aligned}
& \mathbf{P}: \mathbf{B}=108^{\circ} 30^{\prime} \\
& \mathbf{B}: \mathbf{B}=102^{\circ} 26^{\prime} \\
& \mathrm{O}: \mathbf{B}=82^{\circ} 58^{\prime} \quad \text { (annkhernd) } \\
& \mathbf{P}: \mathbf{P}=64^{\circ} 2^{\prime} \\
& \mathbf{B}: \mathbf{B}=68^{\circ} 9^{\prime} .
\end{aligned}
$$

Die einfachsten Krystalle (Fig. 1) entsprechen der Combination $\infty$ P.OP, tafelförmig durch Vorwalten von OP (B). Bei den gröfseren Krystallen stellt sich dazu die positive Hemipyramide (S) ein, welche jedoch stots hemizdrisch nem an der rückwärtigen Halfte der Krystalle ausgebildet ist (Fig. 2). Durch Verschwinden der rückwärtigen Hälfte des Prismas und Vergröfserung der Pyramidenflächen $\mathbf{S}$ entstehen die Krystalle, welche Fig. 3 darstellt. Die dünnen tafelförmigen Krystalle (Fig. 4) entsprechen der Combination 0P. $\infty \mathrm{P} \cdot \frac{\mathrm{P}}{2} \cdot 2 \mathrm{P} \infty$. Das Orthodoma $2 \mathrm{P} \infty(\mathrm{O})$ ist selten scharf ausgebildet. Die Flächen $S$ sind parallel der Combinationskante mit B feingestreift. Spaltbarkeit ondeutlich. Die Ebene der optischen Axen liegt im klinodiagonalen Hauptschnitt, dio Mittellinie fast rechtwinkelig auf $0 P$. Die dünntafelförmigen Krystalle zeigen im Polarisationsmikroscop das Lemniscatensystem vollständig. *

Das unterphosphorsaure Natron löst sich erst in $45 \mathrm{Th}$. kalten, aber schon in $5 \mathrm{Th}$. kochenden Wassers auf; die Lósung reagirt sauer; deshalb und wegen seiner Zusammensetzung ist dieses Salz als saures Salz zu bezeichnen.

Es verliert bei gelindem Erwärmen sein Krystallwasser, fângt dann an zu spratzen, indem der Wasserstoff des Constitutionswassers beim Entweichen sich entzündet, während der Sauerstoff mit der Unterphosphorsăure sich zu Metaphosphorsäure vereinigt :

$$
\mathrm{NaOHO}_{\mathrm{OHO}}=\mathrm{NaOPO}_{8}+\mathrm{H} \text {. }
$$

Dabei tritt nur zuweilen ein schwacher Geruch nach 
Phosphorwasserstoff auf und es bleibt metaphosphorsaures Natron als glasartige Masse zurück. Wird das bei $100^{\circ} \mathrm{C}$. getrocknete Salz allmälig ouf $200^{\circ} \mathrm{C}$. erhitzt, so erleidet es weiter keinen Gewichtsverlust, schmilzt dann hei circa $250^{\circ}$ und wird erst bei Rothglühhitze langsam zersetzt, indem eine teigartige Masse entsteht, aus welcher von Zeit zu Zeit brennender Wasserstoff entweicht; der Rückstand enthält Phosphornatrium. Wird das unterphosphorsaure Natron im Kohlensinurestrom erhitzt, so ist keine Feuererscheinung wahrnehmbar und das entweichende Gas ist geruchlos, also frei von Phosphorwasserstoffgas.

Das Salz ist an der Luft bei gewöhnlicher Temperatur vollkommen unveränderlich, es verliert weder Krystallwasser, noch geht es in phosphorigsaures oder phosphorsaures Salz über; von Alkohol wird es nicht gelöst, sondern in Folge von Wasserentziehung undurchsichtig; ebenso ron concentrirter Schwefelsãure; aber in verdünnter Schwefelsäure ist es leichter löslich, als in Wasser; es löst sich leicht in Salmiakgeist, langsam in Sodalösung auf. Die Auflösung in Wasser ist luftbeständig.

Zur Ausführung der Analyse bestimmte ich zuerst den Gehglt an Krystallwasser, welcher bei Sakz von verschiedenen Darstellungen zwiṣchen 34,25 pC. und 34,95 pC. schwankte; die oben gegebene Formel verlangt $34,39 \mathrm{pC}$. Den bei stärkerem Erhitzen entweichenden Wasserstoff durch den Gewichtsverlust $\mathrm{zu}$ bestimmen, erfordert empfindlichere $\mathrm{W}$ agen, als mir zur Verfügung stehen; ich fand circa $1 \mathrm{pC}$., während die Formel nur 0,63 pC. verlangt; doch ist ein geringes Entweichen von Phosphorwasserstoff bei der grofsen Differenz der Atomgewichte von grofsem Einflufs auf das Resultat. "Die Sauerstoffmenge, welche das Salz gebraucht, um in phosphorsav'es Natron übergeführt zu werden, ist mittelst übermangansauren Kalis leicht und sicher zu bestimmen, wenn man 
wie bei dem Austitriren der freien Säure die Flüssigkeit gelinde erwärmt und das Chamäleon möglichst rasch zufügt; meine Versuche schwankten zwischen 4,95 und 5,02 pC. Sauerstoff, während die obige Formel $5,09 \mathrm{pC}$. Sauerstoff für die vollkoinmene 0xydation erfordert.

Die Bestimmung des Nutrons kann durch Behandlung der Salzlösung mit kohlensaurem Baryt, noch besser aber durch Fällung mit Bleisalz geschehen, indem damit die Phosphorbestimmung $\mathrm{zu}$ vereinigen ist; das salpetersaure Bleioxyd eignet sich weniger gut hierzu, weil es grofse Neigung hat, in den Niederschlag einzugehen und wur durch langwieriges Auswaschen entfernt werden kann. Basisch- oder neutrales essigsaures Bleioxyd erzeugen in der Auflösung des unterphosphorsauren Natrons denselben Niederschlag von unterphosphorsaurem Bleioxyd, welches in der freiwerdenden Essigsäure durchaus unlöslich ist; nach der Ausfällung des überschüssigen Bleis mittelst Schwefelwasserstoff wird das essigsaure Natron durch schwaches Glühen in kohlensaures Natron übergetührt und als solches gewogen.

Auf diese Weise erhielt ich ans 0,250 Grm. unterphosphorsanrem Natron 0,082 kohlensaures Natron, welohe 0,048 Natron enthalten, entsprechend 19,2 pC., während die Rechnung 19,74 pC. verlangt. 1,000 Grm. unterphosphorsaures Natron mittelst salpetersaurem Bleioxyd suggefullt lieferte mir 0,456 salpetersaures Natron oder 0,1986 Natron, also 19,86 pC. Natron, und 1,821 Grm. unterphosphorsanres Bleioxyd, welches nach der Formel 2 PbOPO, zusammengesetzt ist und demneoh 0,1973 Phosphor enthalt, genau so viel als die Rechnung verlangt.

Die Bestimmung des Phosphors als Phosphorsäure aus dem geglühten Salz lieferte mir kein befriedigendes Resultat $(16,8 \mathrm{pC}$.), indem bei dem nothwendigen Kochen des Glührūckstandes mit Schwefelsāure der Zeitpunkt nicht festzustellen ist, wo sämmtliche Meta- oder Pyrophosphorsäure umgewandelt jst. Ich zog daher vor, das Salz mittelst übermangansauren Kalis in phosphorsaures Salz überzuführen, die 
Phosphorsäure mit Molybdänsäure abzuscheiden und als pyrophosphorsaure Magnesia zu wägen.

Auf diese Weise erhielt ich ans 0,200 Grm unterphosphorsaurem Natron 0,140 pyrophosphorsaure Magnesia, entsprechend 0,0391 Phosphor $=19,55$ pC. Phosphor. Die weniger genaue Methode der Abscheidung des Margans mittelst Schwefelammonium gab 20,17 pC. Phosphor.

\begin{tabular}{lrr} 
& \multicolumn{2}{c}{ Berechnet } \\
\cline { 2 - 3 } $\boldsymbol{N a O}$ & 31 & 19,74 \\
$\boldsymbol{H}$ & 1 & 0,63 \\
$\boldsymbol{P}$ & 8 & 5,09 \\
$\boldsymbol{O}_{4}$ & 31 & 19,74 \\
$\mathbf{G} \mathrm{HO}$ & 32 & 20,40 \\
& $\mathbf{5 4}$ & 34,39 \\
\cline { 2 - 3 } & 157 & $100,00$.
\end{tabular}

\begin{tabular}{|c|c|c|c|}
\hline \multicolumn{4}{|c|}{ Gefundon } \\
\hline 19,20 & 19,86 & - & - \\
\hline 1,00 & - & - & - \\
\hline$\overline{19,20}$ & $\overline{19,73}$ & $\overline{19,86}$ & $=$ \\
\hline $34 \overline{25}$ & $3 \overline{4,37}$ & $\overrightarrow{34,63}$ & 34,95 \\
\hline
\end{tabular}

Bei der Oxydation aufgenommen :

$\begin{array}{lllll}0 & 8 & 5,09 & 5,02 & 4,95 .\end{array}$

Da das unterphosphorsaure Natron, wenn es nicht genügend durch Umkrystallisiren gereinigt worden ist, mit phosphorigsaurem und phosphorsaurem Natron verunreinigt sein kann, will ich über seine Prüfung Folgendes bemerken. Das gepulverte Salz darf sich bei gewöhnlicher Temperatur in der vierzigfachen Menge Wasser nicht vollständig auflösen. Die durch Schütteln des Salzes mit wenig Wasser erhaltene Flüssigkeit darf mit der gebrãuchlichen Molybdänlösung längere Zeit stehen gelassen keinen gelben Niederschlag erzeugen; ist die Molybdänlōsung mit Salzsāure angesäuert, so darf die Flüssigkeit gekocht worden und erst dann die Phosphorsäurereaction geben, wenn einige Tropfen Salpetersäure zugefügt werden; ein anderer Theil der Salzlösung mufs mil ein oder zwei Tropfen Silberlösung versetzt einen weifsen Niederschlag geben, der auch bei Kochhitze schneeweifs bleibt; ich fand diese Reaction empfindlicher, als etwa mit Ammeniak im Ueberschufs zu versetzen und zu erwärmen. Man kann auch die Salzlösung mit kohlensaurem Baryt digeriren, wobei ein Theil der phosphori- 
gen Säure gelöst bleibt und leicht nachweisbar ist; ein anderer Theil wird dann bei der successiven Behandlung des ausgowaschenen Rückstands mit kleinen Mengen Schwefelsăure zuerst abgeschieden; zuletzt erscheint dabei die Phosphorsäure. Ich habe auf diese Weise nachgewiesen, dafs das unterphosphorsaure Natron, wie anfangs zu vermuthen, nicht etwa ein Doppelsalz von phosphorigsuurem und phosphorsaurem Natron ist.

Die Behandlung mit kohlensaurem Baryt ist auch das einzige Mittel, die drei Säuren des Phosphors nachzuweisen, wenn sie mit anderen Säuren vermischt in geringen Mengen nebeneinander vorkommen.

$$
\begin{gathered}
\text { Neutrales unterphosphorsaures Natron, } \\
\mathrm{PO}_{4}, 2 \mathrm{NaO}+10 \mathrm{HO} \text { oder } \mathrm{P}_{9} \mathrm{Na}_{2}+5 \mathrm{H}_{\mathbf{z}} \mathrm{O} .
\end{gathered}
$$

Wird die Auflösung des sauren unterphosphorsauren $\mathrm{Na}-$ trons mit kohlensaurem Natron neutralisirt, so gebraucht man dazu auf ein Aequivalent des ersteren nur etwa die einem Drittel Aequivalent des letzteren entsprechende Menge und man erhält durch Eindanupen nadelförmige Krystalle, welche in Wasser ziemlich leicht löslich sind, beim Trocknen sehr rasch verwittern, aber beim Betrachten unter dem Mikroscop nicht als gleichförmig erscheinen, auch bein Umkrystallisiren wieder von dem sauren Salze liefern.

Werden jedoch gleiche Gewichtstheile beider Saize aufgelöst, was annähernd auch gleichen Aequivalentmengen entspricht, und die Lösung nur wenig eingedampft, so scheiden sich feine seideglänzende federartige Krystalle aus, welche offenbar neutrales unterpbosphorsaures Natron sind. Duroh Pressen zwischen Fliefspapier rasch getrocknet sind sio luftbeständig, erscheinen unter dem Mikroscop als sechsseitige Săulen, verlieren beim Erwärmen viel Krystallwasser und gehen dann bei stärkerem Erhitzen ohse Feuererscheinung 
unter Verbreitung von etwas Phosphorgeruch in eine weifse unaurchsichtige Masse über, welche stellenw eise röthlich gefärbt ist und beum Auflösẹn in Wasser eine trübe gelblichrothe Flüssigkeit giebt.

Dieses Salz enthält also kein Constitutionswasser.

Das neutrale unterphosphorsaure Natron löst sich in ungefähr der dreifsigfachen Menge kaltem, viel leichter in heifsem Wasser auf; die Lösung reagirt schwach alkalisch, liefert aber beim Eindainpfen nicht wieder die ursprünglichen Krystalle, sondern ein Gemisch von diesen mit nadel- und tafelförmigen Krystallen des sauren und wahrscheinlich anch noch eines dritten Salzes (des leicht verwitterbaren). Da das Salz also nicht von seiner Mutterlauge vollständig zu trennen ist, habe ich es nur durch Pressen zwischen Fliefspapier möglichst gereinigt und dann den Krystallwassergehalt und die Menge des aufnehmbaren Sauerstoffs bestimmt.

0,606 Grm. Salz hinterliefsen bei $100^{\circ} \mathrm{C}$. getrocknet 0,345 Grm., batten also 0,260 Wasser oder $42,97 \mathrm{pC}$. verloren.

$0,517 \mathrm{Grm}$. Salz entfärbten $60 \mathrm{CC}$. einer Lösung von übermangansaurem Kali, von welcher 1 CC. entsprach 0,0003 Sauerstoff, sie nahmen also 0,018 Grn. oder 3,67 pC. Sauerstoff auf.

Die hiernach wohl schon festzustellende Formel des Salzes ist $2 \mathrm{NaO} \cdot \mathrm{PO}_{4}+10 \mathrm{HO}$ odor $\mathrm{Na}_{2} \mathrm{PO}_{3}+5 \mathrm{H}_{8} \theta_{1}$ welche 41,8 pC. Krystallwasser und 3,71 pC. anfnehmbaren Sauerstoff verlangt.

\section{Unterphosphorsaures Bleioxyd, $2 \mathrm{PbO}_{\mathrm{PO}} \mathrm{PO}_{4}$ oder Pb.P. $\mathrm{\theta}_{3}$.}

Wie schon bemerkt giebt das saure unterphosphorsaure Natron mit neutralem wie basischem essigsaurem Bleioxyd Niederschläge, welche in ihrer Zusammensetzung identisch sind, d. h. immer auf ein Aequivalent Säure 2 Aequivalente Bleioxyd enthalten und schon beim Trocknen an der Luft vollkommen wasserfrei werden.

Das unterphosphorsaure Bleioxyd ist ein sehr weifses 
PuIver, unlöslich in Wísser, verdünnter Essigsăure und Unterphosphorsäure, aher löslich in verdūnnter Salpetersâure ; trotzdem kann die Unterphosphorsãure auch mittelst salpetersauren Bleioxyds aus dem sauren Natronsalz ausgefällt werden, es ist nur ein grofser Ueberschufs des Fallungsmittels nothwendig; von Schwefelsäure wird das Salz leicht zerlegt.

Das unterphosphorsaure Bleioxyd kann auf $120^{\circ} \mathrm{C}$. erhitzt werden, ohne eine Veränderung zu erleiden, aber bei etwas höherer Temperatur verglimmt es Cohne dafs Wasser entweicht) zu einem schwarzen Pulver; bei Verbrennung etwas gröfserer Mengen in einem Tiegelchen ist der Rückstand nur auf der Oberfläche schwarz, wo der Sauerstoff der Luft sofort in der zur volkommenen Oxydation nöthigen Menge vorhanden ist. Die unteren Schichten aber sind grau oder weifslich; dann ist auch ein schwacher Phosphorgeruch bei dem Verbrennungsprocefs bemerkbar. Deshalb stimmt auch die Gewichtszunahne in Wirklichkeit nicht mit der Rechnung genau überein; dieselbe betrug bei einem Versuche für 1,705 Grm. unterphosphorsaures Bleioxyd 0,035 Grm., während die Formel $2 \mathrm{PbO} \cdot \mathrm{PO}_{4}+\mathrm{O}=2 \mathrm{PbO} . \mathrm{PO}_{5}$ eine Gewichtszunahme von $0,047 \mathrm{Grm}$. verlangen würde. H. Rose hat die schwarze Masse, welche bei der Erhitzung des phosphorigsauren Bleioxyds zurückbleibt, analysirt und die Formel $6 \mathrm{PbO} 2 \mathrm{PO}_{5}$ für sie gefunden.

Der Rückstand löst sich erst nach längerem Kochen in Salpetersäure vollständig auf.

Die Analyse des unterphosphorsauren Bleioxyds führte ich in der Weise aus, dafs ich die Menge der Saure mittelst übermangansauren Kalis in dem mil verdünnter Schwefelsäure erwärmten Salz bestimmte und in einer anderen Portion des Salzes durch Auflösen in Salpetersăure, Fällung mittelst Schwefelsäure u. s. w. das Bleioxyd als schwefelsaures Bleioxyd wog. 
0,100 Grm. unterphosphorsaures Bleioxyd verlangten z. B. 9,4 CC. einer Chamăloonlösung, von welcher 1 CC. enteprach 0,00029 Sauerstoff, also im Ganzen 0,00273 Sauerstoff, whhrend die Rechnung (286:8) oine Sauerstoffaufnahme ron 0,00279 orfordert; diese 0,00273 Sauerstoff entsprechen 0,02149 Unterphosphorsäure, was also 21,49 Theilen Unterphosphorsaure in 100 Theilen Salz entspricht.

1,050 Grm. unterphosphorsaures Bleioxyd lieferten 1,101 schwefelsaures Bleioxyd, welches 0,810 Bleinxyd enthult und einem Procentgohalt von 77,14 Bleioxyd entspricht.

\begin{tabular}{lrrr}
2 & \multicolumn{2}{c}{ Berechnet } & Gefunden \\
\cline { 2 - 4 } & 223 & 77,97 & 77,14 \\
$\mathrm{PO}_{4}$ & 63 & 22,03 & $21,49$.
\end{tabular}

Ich bin eben mit der Reindarstellung anderer unterphosphorsaurer Salze beschäftigt und werde später über das $\mathbf{E r}-$ gebnifs dieser Untersuchungen berichten; ich will deshalb anch vorerst noch keine Vermuthungen über die wahrscheinliche Constitution der Unterphosphorsäure aussprechen. Mittheilungen über Versuche zur synthetischen Darstellung der Unterphosphorsäure durch Wechselwirkung zwischen phosphoriger Sãure und Phosphorsäure, resp. deren Derivaten *), sowie zur Ermittelung der günstigsten Bedingungen für ihre Bildung bei der Oxydation des Phosphors behalte ich gleichfalls einer zweiten Abhandlung vor.

W or ms, März 187 .

*) Ich habe Unterphosphorsäure in einer von Merck in Darmstadt bezogenen phosphorigen saure gefunden. Letztere gab anfange keine, später aber sehr starke Salzshurereaction; vermuthlich entbielt dieselbe ein Oxychlorid, welches durch Wusser in Unterphosphorsäure und Balzstlure zerfallt. 
Liebriys Annalen der Chemie Bd.187 Taf III.

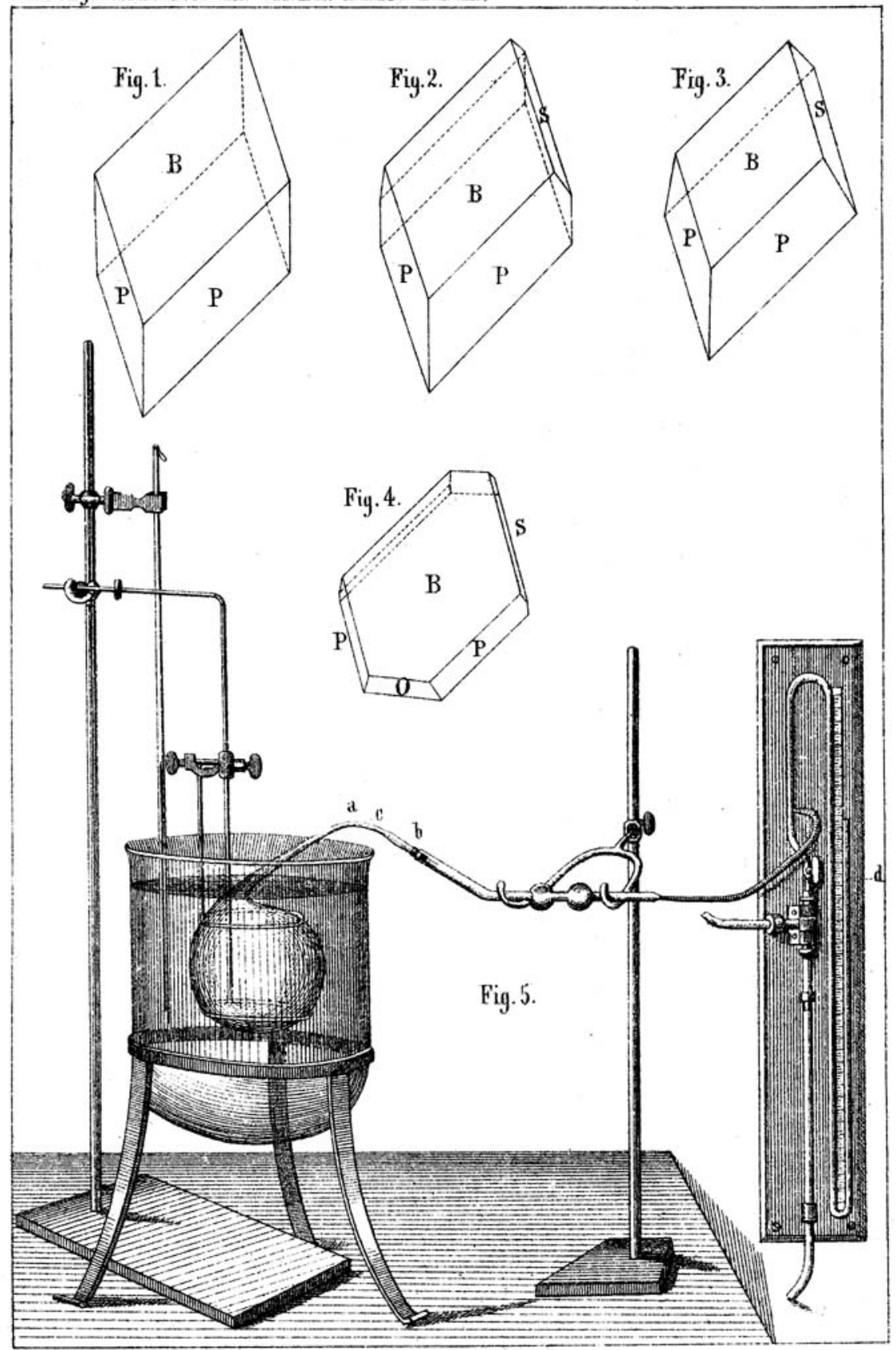

